\title{
The Poisson equation in density fitting for the Kohn-Sham Coulomb problem
}

\author{
F. R. Manby ${ }^{\mathrm{a})}$ \\ School of Chemistry, University of Bristol, Cantock's Close, Bristol BS8 1TS, United Kingdom \\ P. J. Knowles ${ }^{\text {b) }}$ and A. W. Lloyd \\ School of Chemistry, University of Birmingham, Edgbaston, Birmingham B15 2TT, United Kingdom
}

(Received 18 July 2001; accepted 10 September 2001)

\begin{abstract}
A new density fitting approach to the Coulomb problem in Kohn-Sham and Hartree-Fock theory is introduced. Almost all of the 2- and 3-index repulsion integrals become simple overlap-like integrals, without approximation. The method is tested on numerous benchmark problems, which reveal that accuracy equal to or better than standard density fitting can be achieved with the evaluation of around a tenth of the number of Coulomb integrals. The scaling properties of the method are illustrated for polyalanine helices up to Ala 16 . (c) 2001 American Institute of Physics.
\end{abstract}

[DOI: $10.1063 / 1.1414370]$

\section{INTRODUCTION}

A bottleneck in traditional implementations of KohnSham (KS) theory ${ }^{1,2}$ is the evaluation of the electron repulsion integrals (ERIs), necessary for the evaluation of the Coulomb contribution to the Fock matrix. The electronic density in KS and Hartree-Fock (HF) theory is expanded in a product basis,

$$
\rho(\mathbf{r})=\sum_{\mu \nu} \gamma_{\mu \nu} \chi_{\mu}(\mathbf{r}) \chi_{\nu}^{*}(\mathbf{r}),
$$

so the Coulomb energy,

$$
E=\frac{1}{2} \int d \mathbf{r}_{1} \int d \mathbf{r}_{2} \frac{\rho\left(\mathbf{r}_{1}\right) \rho\left(\mathbf{r}_{2}\right)}{r_{12}},
$$

where $r_{12} \equiv\left|\mathbf{r}_{1}-\mathbf{r}_{2}\right|$, has to be constructed from 4-index ERIs. Formally, this is an $\mathcal{O}\left(N^{4}\right)$ computational process, but since the density matrix is sparse, with $\mathcal{O}(N)$ nonzero elements for large molecules, the number of integrals to be evaluated actually scales as $\mathcal{O}\left(N^{2}\right)$. Despite huge advances in integral evaluation technology, these integrals are still time-consuming to compute, and the Coulomb energy evaluation is the main bottleneck for large calculations. It is possible, however, to avoid the evaluation of 4-index ERIs altogether. This is achieved by the introduction of an auxiliary basis $\left\{\Xi_{A}\right\}$ in which one constructs an approximate density,

$$
\check{\rho}(\mathbf{r})=\sum_{A} d_{A} \Xi_{A}(\mathbf{r}) .
$$

Then the Coulomb energy arising from the interaction of $\check{\rho}$ with itself only requires 2-index ERIs, and has the form

$$
\check{E}=\frac{1}{2} \mathbf{d}^{\mathrm{T}} \mathbf{J d},
$$

where

\footnotetext{
${ }^{a)}$ Electronic mail: Fred.Manby@bristol.ac.uk

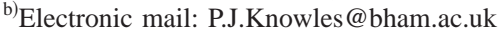

$$
J_{A B}=\int d \mathbf{r}_{1} \int d \mathbf{r}_{2} \frac{\Xi_{A}\left(\mathbf{r}_{1}\right) \Xi_{B}\left(\mathbf{r}_{2}\right)}{r_{12}},
$$

and where $\mathbf{d}$ is the coefficient vector that minimizes the error in the auxiliary density.

There are several ways to measure this error, generally having the form

$$
\Delta=\frac{1}{2} \int d \mathbf{r}_{1} \int d \mathbf{r}_{2}[\rho-\check{\rho}]\left(\mathbf{r}_{1}\right) \hat{W}[\rho-\check{\rho}]\left(\mathbf{r}_{2}\right),
$$

and differing through the choice of the weight operator $\hat{W}$. Least squares fitting-performed by choosing $\hat{W}=\delta\left(r_{12}\right)$-at first appears attractive, since the only 3 -index integrals it involves are overlaps, not Coulomb integrals. However, the performance of least squares fitting has been found to be very unsatisfactory, both by others ${ }^{3}$ and in our own investigations. The weight operator that shows good convergence of energies and other properties with respect to auxiliary basis set is $\hat{W}=r_{12}^{-1}$ leading to the error expression $^{3,4}$

$$
\Delta=\frac{1}{2} \int d \mathbf{r}_{1} \int d \mathbf{r}_{2} \frac{[\rho-\check{\rho}]\left(\mathbf{r}_{1}\right)[\rho-\check{\rho}]\left(\mathbf{r}_{2}\right)}{r_{12}} .
$$

The error is minimized with respect to the coefficients $\mathbf{d}$ in the auxiliary basis by setting $\boldsymbol{\nabla}_{\mathbf{d}} \Delta=\mathbf{0}$, and this leads to the linear equations

$$
\sum_{B} J_{A B} d_{B}=\sum_{\mu \nu} I_{A, \mu \nu} \gamma_{\mu \nu}
$$

where I is a 3-index matrix of ERIs of the form

$$
I_{A, \mu \nu}=\int d \mathbf{r}_{1} \int d \mathbf{r}_{2} \frac{\Xi_{A}\left(\mathbf{r}_{1}\right) \chi_{\mu}\left(\mathbf{r}_{2}\right) \chi_{\nu}^{*}\left(\mathbf{r}_{2}\right)}{r_{12}} .
$$

This fitting of the density considerably reduces the prefactor in the evaluation of the Coulomb contribution to the Fock matrix, but the scaling remains $\mathcal{O}\left(N^{2}\right)$ owing to the long-ranged nature of the Coulomb interaction. To achieve a 
linear-scaling method (either in Coulomb fitting or traditional 4-index integral methods), the asymptotic multipolar form of the Coulomb interaction between pairs of charge distributions can be exploited. ${ }^{5-9}$ The separation of long- and short-range Coulomb effects can be further refined by partitioning the Coulomb potential using an error function, ${ }^{10}$

$$
\frac{1}{r}=\frac{\operatorname{erf}(\omega r)}{r}+\frac{\operatorname{erfc}(\omega r)}{r} .
$$

These methods are all based on analytical six-dimensional integrals, but an alternative set of methods exist in which the Coulomb potential is built on a quadrature grid, prior to numerical integration with the density to obtain the Coulomb energy, or with orbital pairs to obtain the Coulomb contribution to the Fock matrix. ${ }^{11-13}$ The Coulomb potential is given by the three-dimensional integral

$$
v\left(\mathbf{r}_{1}\right)=\int d \mathbf{r}_{2} \frac{\rho\left(\mathbf{r}_{2}\right)}{r_{12}},
$$

which has to be performed at each grid point $\mathbf{r}_{1}$. The Coulomb potential also satisfies the Poisson equation,

$$
\hat{P} v=\rho,
$$

where $\hat{P}=-(4 \pi)^{-1} \nabla^{2}$, and one can solve this differential equation for $v$, avoiding the evaluation of any ERIs at all. However, a comparison of methods for computing the Coulomb potential on a grid revealed that it was more efficient to evaluate the integral of Eq. (11) than to solve the Poisson equation. ${ }^{11}$ Nonetheless in this work we reinvestigate the possibility of using the Poisson equation in Coulomb fitting.

It is worth pointing out that although the exact exchange energy in HF and hybrid KS theories is, like the Coulomb energy, constructed from 4-index ERIs, the sparsity of the density matrix can be exploited to construct an efficient linear-scaling method. Thus the Coulomb problem is a bottleneck even in HF theory, and we expect the current work to be of use in that context as well.

\section{THEORY}

In a previous paper ${ }^{14}$ two of us wrote down a density fitting method based on the Poisson equation. Here we refine the method and approach the problem in a slightly different way. We start off from the integral identity ${ }^{15}$

$$
\int d \mathbf{r}_{2} \frac{\hat{P} f\left(\mathbf{r}_{2}\right)}{r_{12}}=f\left(\mathbf{r}_{1}\right),
$$

which holds exactly for any $f(\mathbf{r})$ that vanishes more quickly than $r^{-1}$ as $r \rightarrow \infty$. Equation (13) can be obtained by inserting the Poisson equation [Eq. (12)] into the Coulomb potential expression in Eq. (11). However, we merely note here that Eq. (13) is an exact relation that implies a means of avoiding the troublesome six-dimensional, long-range Coulomb integrals. To apply the identity we will set up a density fitting method by expanding the auxiliary electronic density $\check{\rho}$ in a set of functions of the form $\hat{P} \Xi_{A}$, which we will call Poisson functions, where $\Xi_{A}$ is a Gaussian (and therefore satisfies the requirement of vanishing at long range faster than $r^{-1}$ ),

$$
\check{\rho}(\mathbf{r})=\sum_{A} d_{A} \hat{P} \Xi_{A}(\mathbf{r}) .
$$

Writing down the Coulomb energy in exactly the standard way [Eq. (2)] we have

$\check{E}=\frac{1}{2} \sum_{A B} d_{A} d_{B} \int d \mathbf{r}_{1} \int d \mathbf{r}_{2} \frac{\left[\hat{P}_{1} \Xi_{A}(1)\right]\left[\hat{P}_{2} \Xi_{B}(2)\right]}{r_{12}}$,

where $\hat{P}_{i}$ acts on functions in the coordinates $\mathbf{r}_{i}$. Inserting Eq. (13) and using the Hermiticity of $\hat{P}$, the energy reduces to

$$
\check{E}=\frac{1}{2} \sum_{A B} d_{A} d_{B} \int d \mathbf{r}_{1} \Xi_{A}(1) \hat{P}_{1} \Xi_{B}(1) .
$$

This equation is interesting because it gives an exact expression for the Coulomb energy of the density $\check{\rho}$ using only short-range three-dimensional integrals, which differ from kinetic energy integrals only by a factor of $(2 \pi)^{-1}$.

There is a catch in all this. Consider a multipole of the density $\hat{P} \Xi_{A}(\mathbf{r})$,

$$
q_{A}^{l m}=\int d \mathbf{r} Y_{l m}(\hat{r}) r^{l} \hat{P} \Xi_{A}(\mathbf{r}) .
$$

The function $\Xi_{A}(\mathbf{r})$ vanishes asymptotically so we can use integration by parts to apply $\nabla^{2}$ to the left, and then since $\nabla^{2} Y_{l m}(\hat{r}) r^{l}=0$ we see that $q_{A}^{l m}=0$ for all $l, m$. In other words the fitted density in Eq. (14) can have no total charge, no total dipoles, and so on. In our earlier paper ${ }^{14}$ we alleviated the first problem-that of the vanishing charge-by considering the electrons and nuclei of a neutral system simultaneously. Here we generalize the method by considering the addition of a small number of ordinary basis functions to the expansion in Eq. (14). These functions serve to describe the total charge and higher multipoles of $\check{\rho}$, and to give some gross approximation to the density. The Poisson functions serve to move charge around and produce an accurate model density.

We will now set up density fitting in a basis of $m_{C}$ standard and $m_{P}$ Poisson functions, following Eqs. (4)-(9). The Coulomb matrix $\mathbf{J}$ now blocks into three types of integrals (see Fig. 1), having in the integrand zero, one and two instances of the Poisson operator. These integrals are respectively standard Coulomb integrals, standard overlaps, and the scaled kinetic energy-like integrals of Eq. (16). The 3-index integrals block into $m_{C} m(m+1) / 2$ Coulomb integrals and $m_{P} m(m+1) / 2$ overlaps, where $m$ is the size of the atomic orbital basis. Since $m_{C}$ is small, by far the greater proportion of the 2- and 3-index integrals are short-ranged, and the $S$ and $P$ blocks in Fig. 1 are sparse.

Once the Coulomb matrices in the mixed basis have been set up, density fitting can be performed in exactly the normal way.

\section{BASIS SETS AND TEST CASES}

To test the method, it is necessary to optimize mixed basis sets. For the preliminary calculations presented here, 

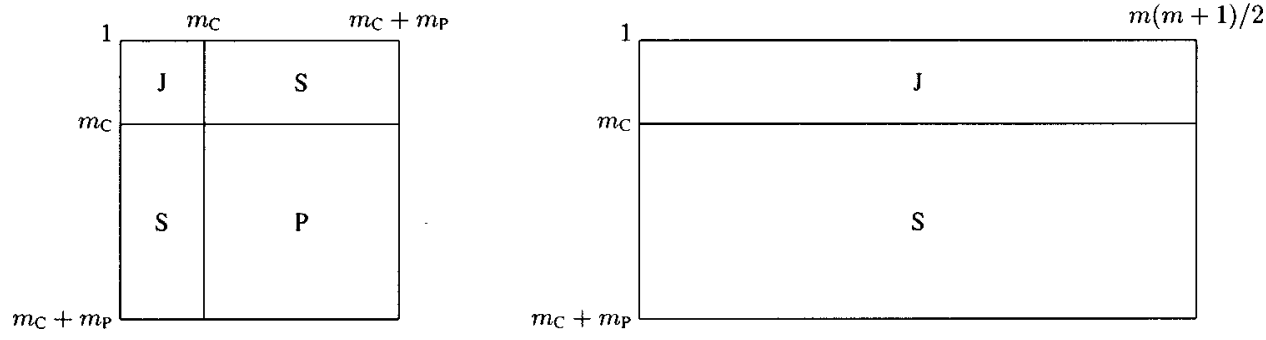

FIG. 1. Blocking of 2- and 3-index integrals in mixed Poisson density fitting. The $\mathrm{AO}$ basis is of size $m$ and there are $m_{C}$ and $m_{P}$ standard and Poisson fitting functions, respectively. The blocks are labeled $J$ (standard Coulomb integrals), $S$ (standard overlap integrals), and $P$ (scaled kinetic energy integrals). we optimize basis sets for the elements of the first row of the periodic table. Our procedure for doing so closely follows that of Eichkorn et al., ${ }^{16}$ and we choose to use their 3-parameter extension of even-tempered series of exponents [Eq. (27) of Ref. 16]. The optimizations are performed using a Powell minimizer from several starting guesses. The regular way in which the optimal parameters vary across the periodic table makes the optimizations increasingly easy.

Our procedure for optimizing a basis for a given atom was as follows:

(1) Optimize a minimal basis of standard s-type functions for the isolated atom;

(2) Optimize Poisson functions ( $s$ and in some cases $d$-type functions) for the isolated atom;

(3) Simultaneously optimize a single, standard $p$ function and a set of Poisson $p, f$, and possibly $d$ functions for the hydride.

The size of bases was chosen to obtain errors in the Coulomb energy below $50 \times 10^{-6}$ hartree for atoms and 0.2 $\times 10^{-3}$ hartree per atom for molecules. The basis sets for $\mathrm{C}$, $\mathrm{N}, \mathrm{O}$, and $\mathrm{F}$ have $2 s 1 p$ functions contracted to $1 s 1 p$ for the standard basis and $6 s 3 p 6 d 1 f$ for the Poisson basis. These

TABLE I. LDA bond lengths, dipole moments, and harmonic frequencies of ground states of first-row diatomics using an exact Coulomb treatment and the approximation introduced in this work. Average errors are provided in the final row.

\begin{tabular}{ccccccccc}
\hline \hline & \multicolumn{2}{c}{$r /$ bohr } & & \multicolumn{2}{c}{$\left|\mu_{z}\right| / \mathrm{D}$} & & \multicolumn{2}{c}{$\nu / \mathrm{cm}^{-1}$} \\
\cline { 2 - 3 } \cline { 8 - 9 } \cline { 8 - 9 } Diatom & Exact & This work & & Exact & This work & & Exact & This work \\
\hline $\mathrm{H}_{2}$ & 1.4774 & 1.4773 & & 0.0000 & 0.0000 & & 4153.30 & 4153.13 \\
$\mathrm{LiH}$ & 3.0756 & 3.0762 & & 5.5546 & 5.5531 & & 1343.19 & 1342.34 \\
$\mathrm{BeH}$ & 2.5998 & 2.5993 & & 0.0827 & 0.0785 & & 1943.49 & 1941.37 \\
$\mathrm{BH}$ & 2.4240 & 2.4243 & & 1.3888 & 1.3888 & & 2184.34 & 2183.39 \\
$\mathrm{CH}$ & 2.1369 & 2.1369 & & 1.0614 & 1.0616 & & 2867.46 & 2867.91 \\
$\mathrm{NH}$ & 2.0138 & 2.0136 & & 1.5449 & 1.5428 & & 3127.87 & 3126.78 \\
$\mathrm{OH}$ & 1.9160 & 1.9156 & & 1.9316 & 1.9289 & & 3293.75 & 3294.91 \\
$\mathrm{HF}$ & 1.7671 & 1.7671 & & 1.8449 & 1.8443 & & 3945.76 & 3943.16 \\
$\mathrm{LiF}$ & 2.9778 & 2.9781 & & 5.5384 & 5.5378 & & 977.19 & 977.07 \\
$\mathrm{BeF}$ & 2.6545 & 2.6542 & & 1.0549 & 1.0551 & & 1237.80 & 1236.15 \\
$\mathrm{BN}$ & 2.5213 & 2.5207 & & 1.4384 & 1.4384 & & 1534.69 & 1533.50 \\
$\mathrm{BO}$ & 2.2921 & 2.2916 & & 1.8272 & 1.8292 & & 1872.72 & 1871.08 \\
$\mathrm{CN}$ & 2.2310 & 2.2308 & & 0.9027 & 0.8989 & & 2130.56 & 2130.75 \\
$\mathrm{CO}$ & 2.1523 & 2.1520 & & 0.3089 & 0.3109 & & 2160.47 & 2160.99 \\
$\mathrm{~N}_{2}$ & 2.0981 & 2.0975 & & 0.0000 & 0.0000 & & 2397.18 & 2399.60 \\
$\mathrm{NO}$ & 2.1860 & 2.1856 & & 0.1850 & 0.1842 & & 1964.61 & 1964.81 \\
$\mathrm{NF}$ & 2.4493 & 2.4485 & & 0.6983 & 0.7010 & & 1239.12 & 1240.76 \\
$\mathrm{O}_{2}$ & 2.2855 & 2.2850 & & 0.0000 & 0.0000 & & 1628.67 & 1629.32 \\
$\mathrm{~F}_{2}$ & 2.6368 & 2.6366 & & 0.0000 & 0.0000 & & 1033.21 & 1032.03 \\
\hline Avg. error & & 0.0004 & & 0.0012 & & 1.1 \\
\hline \hline
\end{tabular}

bases are somewhat larger than those used in standard density fitting, but the integral evaluation is easier, and, as we shall show, the number of integrals to compute is much smaller.

To test the accuracy of the method, we have computed the bond lengths, dipole moments and harmonic frequencies of the ground states of 19 first-row diatomics. This and all tests are based on comparisons between LDA calculations ${ }^{17}$ using the cc-pVDZ atomic orbital basis sets of Dunning. ${ }^{18}$ The results and average errors are given in Table I. The errors in bond lengths and dipole moments are consistently extremely small, and the frequencies are generally reproduced to within $1 \mathrm{~cm}^{-1}$.

Our second test examines the accuracy of the method in computing energy differences of different magnitudes. We therefore compute the dissociation energy (using fixed geometries) of benzene into three acetylene molecules, the singlet-triplet splitting of methylene and the rotational barrier of ethane. The values and errors are given in Table II. Although the relative errors do increase as the quantity being computed decreases, the smallest energy - the rotational barrier of ethane-is in error by only $0.8 \%$ in the current method.

A further energy difference that provides a test of the method is that between the zwitterionic and neutral forms of glycine. Table III shows results using an exact Coulomb treatment, standard density fitting with the basis sets of Eichkorn et al. ${ }^{16}$ and the current method. The performance is

TABLE II. Energy differences of three different magnitudes computed using an exact Coulomb treatment and this work. The three cases are the dissociation of benzene into three acetylene molecules, the singlet/triplet splitting of methylene, and the rotational barrier of ethane. The errors range between $0.07 \%$ and $0.8 \%$ of as the size of the computed quantity decreases.

\begin{tabular}{rrrl}
\hline \hline & \multicolumn{2}{c}{ E/hartree } & \\
\cline { 2 - 3 } & \multicolumn{1}{c}{ Exact } & This work & Error/10 $0^{-3}$ hartree \\
\hline $\mathrm{C}_{6} \mathrm{H}_{6} \rightarrow 3 \mathrm{C}_{2} \mathrm{H}_{2}$ & & & \\
$\mathrm{C}_{6} \mathrm{H}_{6}$ & -230.096551 & -230.097085 & 0.53 \\
$\mathrm{C}_{2} \mathrm{H}_{2}$ & -76.588225 & -76.588328 & 0.10 \\
Dissociation & 0.331876 & 0.332103 & 0.23 \\
& & & \\
Singlet/triplet $\mathrm{CH}_{2}$ & & & \\
Singlet & -38.704500 & -38.704640 & 0.14 \\
Triplet & -38.743226 & -38.743307 & 0.081 \\
Splitting & 0.038726 & 0.038667 & 0.059 \\
& & & \\
Barrier of $\mathrm{C}_{2} \mathrm{H}_{6}$ & & & 0.44 \\
Eclipsed & -79.024833 & -79.025269 & 0.39 \\
Staggered & -79.030755 & -79.031147 & 0.045 \\
Barrier & 0.005922 & 0.005877 & \\
\hline \hline
\end{tabular}


TABLE III. Energies and dipole moments of zwitterionic and neutral forms of glycine using an exact Coulomb treatment, standard density and the method of this work, along with the errors in computed values. Clearly the energy difference between the two forms is treated more accurately in the current work than in standard density fitting, and the dipoles in the two approaches are roughly equal in accuracy.

\begin{tabular}{lccc}
\hline \hline & Exact & Standard & This work \\
\hline Zwitterion & & & \\
$E /$ hartree & -282.149160 & -282.149708 & -282.149712 \\
$\mu_{x} /$ bohr & -3.6497 & -3.6468 & -3.6484 \\
$\mu_{y} /$ bohr & -1.2700 & -1.2689 & -1.2697 \\
$\mu_{z} /$ bohr & 1.3851 & 1.3848 & 1.3839 \\
& & & \\
Neutral & & & \\
$E /$ hartree & -282.144421 & -282.145094 & -282.144971 \\
$\mu_{x} /$ bohr & 1.4777 & 1.4771 & 1.4794 \\
$\mu_{y} /$ bohr & -1.0268 & -1.0257 & -1.0258 \\
$\mu_{z} /$ bohr & -0.1293 & -0.1292 & -0.1297 \\
\hline$\Delta E / \mathrm{kJ} \mathrm{mol}^{-1}$ & 12.442 & 12.115 & 12.448 \\
\hline \hline
\end{tabular}

very good (less than $0.05 \%$ error in the isomerization energy), and, perhaps fortuitously, significantly better than that obtained with the standard fitting basis of Ref. 16. The results also show that molecular properties such as the dipole moment, which provide a more stringent test of the faithfulness of the density fitting in regions of space not strongly weighted in the fitting error functional, can be reproduced with similar accuracy in the current and standard methods.

Our final set of tests regard the number of integrals computed in the current method compared to standard density fitting methods. We consider polyalanine helices with up to 16 amino acids, and count the number of primitive 3 -index integrals that have to be computed in the standard and current density fitting approaches. Integrals are screened with a threshold of $10^{-8}$ hartree. The results are shown in Fig. 2. The number of Coulomb integrals evaluated in the current method remains consistently around $10 \%$ of the number in standard Coulomb fitting, and the number of 3-index overlaps rises only linearly. Even for the smallest (4 peptide) molecule, the total number of integrals is less than with the standard basis, and the vast majority of these are of the $S$ type, rather than the more expensive $J$. Since the number of

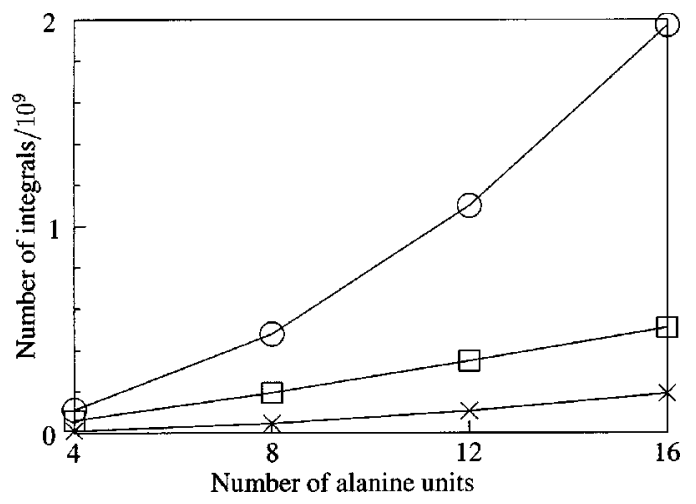

FIG. 2. Number of 3-index integrals in density fitting calculations on polyalanine helical peptides. The lines are the number of Coulomb integrals in standard density fitting (circles) and in the current work (crosses), and the number of 3-index overlap integrals in the current work (squares). non-zero $S$ integrals grows only linearly with system size, for the largest $\left(\mathrm{Ala}_{16}\right)$ system, the integral evaluation problem is considerably reduced, and we are still in the regime where the $\mathcal{O}\left(N^{2}\right)$ number of $J$ integrals is less than the $\mathcal{O}(N)$ number of $S$ integrals.

\section{DISCUSSION}

We have introduced a new density fitting method for the Coulomb problem in KS and HF theory. Most of the functions in the auxiliary basis having the form $\hat{P} \Xi_{A}(\mathbf{r})$, and the Coulomb integrals involving one or two of these functions become exactly equivalent to simple overlap-like integrals. This allows us to reduce the number of Coulomb integrals in density fitting by a factor of around 10. A small number of standard functions is required in the auxiliary basis since the functions $\hat{P} \Xi_{A}(\mathbf{r})$ contain no charge and have vanishing multipoles. They do, however serve to move density around the molecule to provide an accurate auxiliary density. Further savings can be made. Since the standard basis in this method is only present to give a very rough approximation to the density, we will reoptimize the basis sets using the same exponent for the more diffuse $s$ function and the $p$ function. Since none of the functions in the standard basis needs to be diffuse, the multipole approximation will rapidly take over, and a very small number of true Coulomb integrals will have to be evaluated.

For very large systems, the bottleneck in standard density fitting is the dense linear algebra need to form $\mathbf{d}$. In the current method only the $J$-block of the Coulomb matrix (see Fig. 1) is dense, and this amounts only to around $1 \%$ of the whole matrix. Therefore even for large systems, the linear algebra to be performed is effectively sparse. Naturally there comes a point where the dense part of the matrix becomes too large, but the current method delays that threshold until the system is roughly ten times larger.

Despite the great savings in terms of the number of integrals to be computed, and the fact that most of them are overlap integrals, the method is accurate. Extensive tests on energies, energy differences, bond lengths, dipoles, and harmonic frequencies reveals that the current method is equal or superior in accuracy to standard density fitting methods. Also accuracy can be increased by the addition of further Poisson functions at relatively little cost, as these only incur the evaluation of overlap integrals, almost all of which vanish.

\section{ACKNOWLEDGMENTS}

The authors would like to thank Dr. John Wilkie for providing the coordinates for the alanine helices, and one of the authors (F.R.M.) is grateful to the Royal Society for financial support.

\footnotetext{
${ }^{1}$ W. Kohn and L. J. Sham, Phys. Rev. A140, 1133 (1965).

${ }^{2}$ R. G. Parr and W. Yang, Density-Functional Theory of Atoms and Molecules (Oxford University Press, Oxford, 1989).

${ }^{3}$ O. Vahtras, J. Almlöf, and M. W. Feyereisen, Chem. Phys. Lett. 213, 514 (1993).

${ }^{4}$ B. I. Dunlap, Phys. Chem. Chem. Phys. 2, 2113 (2000).

${ }^{5}$ L. Greengard and V. Rokhlin, J. Comput. Phys. 73, 325 (1987).

${ }^{6}$ L. Greengard, Science 265, 909 (1994).
} 
${ }^{7}$ C. A. White, B. G. Johnson, P. M. W. Gill, and M. Head-Gordon, Chem. Phys. 230, 1 (1994).

${ }^{8}$ M. C. Strain, G. E. Scuseria, and M. J. Frisch, Science 271, 5245 (1996).

${ }^{9}$ P. M. W. Gill, Chem. Phys. Lett. 270, 193 (1997).

${ }^{10}$ J. P. Dombroski, S. W. Taylor, and P. M. W. Gill, J. Phys. Chem. 100, 6272 (1996).

${ }^{11}$ V. Termath and N. C. Handy, Chem. Phys. Lett. 230, 17 (1994).

${ }^{12}$ E. J. Baerends, D. E. Ellis, and P. Ros, Chem. Phys. 2, 41 (1973).

${ }^{13}$ C. Fonseca Guerra, J. G. Snijders, G. te Velde, and E. J. Baerends, Theor.
Chem. Acc. 99, 391 (1998).

${ }^{14}$ F. R. Manby and P. J. Knowles, Phys. Rev. Lett. 87, 163001 (2001).

${ }^{15}$ P. M. Morse and H. Feshbach, Methods of Theoretical Physics (McGrawHill, New York, 1953), Sec. 1.4.

${ }^{16}$ K. Eichkorn, O. Treutler, H. Öhm, M. Häser, and R. Ahlrichs, Chem. Phys. Lett. 240, 283 (1995).

${ }^{17}$ S. J. Vosko, L. Wilk, and M. Nusair, Can. J. Phys. 58, 1200 (1980).

${ }^{18}$ T. H. Dunning, Jr., J. Chem. Phys. 90, 1007 (1989). 